\title{
Temporal Lobe Epilepsy Surgery Failures: A Review
}

\author{
Adil Harroud, ${ }^{1}$ Alain Bouthillier, ${ }^{2}$ Alexander G. Weil, ${ }^{2}$ and Dang Khoa Nguyen' ${ }^{1}$ \\ ${ }^{1}$ Division of Neurology, Hôpital Notre-Dame du CHUM, University of Montreal, Montreal, QC, Canada H2L 4M1 \\ ${ }^{2}$ Division of Neurosurgery, Hôpital Notre-Dame du CHUM, University of Montreal, Montreal, QC, Canada H2L 4M1
}

Correspondence should be addressed to Dang Khoa Nguyen, d.nguyen@umontreal.ca

Received 26 November 2011; Revised 17 January 2012; Accepted 1 February 2012

Academic Editor: Giangennaro Coppola

Copyright ( 2012 Adil Harroud et al. This is an open access article distributed under the Creative Commons Attribution License, which permits unrestricted use, distribution, and reproduction in any medium, provided the original work is properly cited.

\begin{abstract}
Patients with temporal lobe epilepsy (TLE) are refractory to antiepileptic drugs in about $30 \%$ of cases. Surgical treatment has been shown to be beneficial for the selected patients but fails to provide a seizure-free outcome in 20-30\% of TLE patients. Several reasons have been identified to explain these surgical failures. This paper will address the five most common causes of TLE surgery failure (a) insufficient resection of epileptogenic mesial temporal structures, (b) relapse on the contralateral mesial temporal lobe, (c) lateral temporal neocortical epilepsy, (d) coexistence of mesial temporal sclerosis and a neocortical lesion (dual pathology); and (e) extratemporal lobe epilepsy mimicking TLE or temporal plus epilepsy. Persistence of epileptogenic mesial structures in the posterior temporal region and failure to distinguish mesial and lateral temporal epilepsy are possible causes of seizure persistence after TLE surgery. In cases of dual pathology, failure to identify a subtle mesial temporal sclerosis or regions of cortical microdysgenesis is a likely explanation for some surgical failures. Extratemporal epilepsy syndromes masquerading as or coexistent with TLE result in incomplete resection of the epileptogenic zone and seizure relapse after surgery. In particular, the insula may be an important cause of surgical failure in patients with TLE.
\end{abstract}

\section{Introduction}

Despite optimal medical therapy, about a third of patients with partial epilepsy continue to have recurrent seizures $[1,2]$. A randomized clinical trial has shown that surgery is superior to prolonged medical therapy for patients with refractory temporal lobe epilepsy (TLE) [3]. However, surgical treatment of temporal lobe epilepsy still fails to provide a seizure-free outcome in $20-30 \%$ of these patients $[4,5]$. Several studies have addressed this issue and have reported several potential causes for these failures.

The reasons behind surgical failure are multiple and vary from one patient to another, and each pattern of seizure recurrence is likely to have its own explanation. Five major causes are commonly identified in the literature: (1) insufficient resection of mesial temporal structures, (2) insufficient or nonresection of temporal neocortex, (3) dual pathology, (4) relapse on the contralateral temporal lobe, and (5) extratemporal and temporal plus epilepsy. This review will address each of these categories separately and report the proposed underlying mechanisms precluding seizurefree outcomes.

\section{Insufficient Resection of Mesial Structures}

In the large majority of TLE cases, the seizures arise mainly from the mesial temporal lobe [6]. An obvious reason of failure to relieve seizures after surgery is insufficient resection of these epileptogenic structures, whether it is from imprecise delimitation or functional and surgical limitation. Several reports of patients who underwent postsurgical reevaluation concluded that a large part of surgical failures can be attributed to residual epileptogenic mesial temporal structures, and that further resection of these structures can result in a seizure-free outcome in $52-60 \%$ of reoperated cases [7-10].

Wyler et al. [7] reported 37 patients who failed a first epilepsy surgery and underwent reoperation. The original resection was temporal in 16 patients, with 10 (62\%) achieving seizure freedom after a second temporal surgery. In 9 of the 10 seizure-free patients, reoperation consisted in further resection of the hippocampus only; the remaining patient had removal of a structural lesion. The authors concluded that "the most common cause for poor outcome 
of the original operation in patients with TLE was insufficient hippocampal resection."

Similar results were reported by Awad et al. [8], who found that residual epileptogenicity was confined to the residual mesial structures in 6 out of 10 patients with a temporal focus of recurrence. Germano et al. [9] reviewed 40 patients who had first failed anteromedial lobectomy and underwent reoperation. All 40 patients had residual mesial structures, and electrographic abnormalities were confined to the residual ipsilateral temporal lobe in 32 of these patients. The 8 remaining cases had EEG abnormities in multiple areas. Of the 21 patients who became seizure-free after the second operation, 16 had undergone additional resection of residual mesiotemporal structures only. From a total of 262 patients treated surgically for intractable TLE, Salanova et al. [10] identified 41 cases of failure (Engel class III and IV). Postsurgical brain MRI was available for 30 patients and showed evidence of residual mesial temporal structures in $93 \%$ of these patients. Reoperation in 14 of these patients consisted of further resection of the mesial temporal structures alone, with $64 \%$ achieving a seizure-free outcome.

The success of reoperation with further resection of mesial temporal structures clearly indicates that incomplete resection of these structures is a cause of seizure recurrence and surgical failure in a subgroup of patients. However, failure of the second surgery to cure all patients with remnant mesial temporal structures necessarily indicates other possible causes. Furthermore, not all authors agree on the relative importance of this mechanism of failure. While the previously cited studies consider residual mesial temporal structures as the primary reason of surgical failure in TLE, some recent studies believe that other causes may be more important.

Abosch et al. [11] reported that 4 of 7 patients who underwent a second resection of mesial temporal structures became free from seizures. However, the 3 remaining patients did not benefit from the second surgery even if postsurgical MRI showed that resection of the hippocampus, amygdale, and entorhinal cortex was nearly complete. Hennessy et al. [12] found that only $5(25 \%)$ of 20 patients with mesial temporal sclerosis (MTS) who failed a first TLE surgery had MRI evidence of residual hippocampus on the side of surgery. Also, EEG features (scalp EEG for one, intracranial strip electrodes for the other) were supportive of seizures arising in this remnant in only two patients. One of the two was operated and became seizure-free. Ojemann [13] found that only 4 of 19 patients who underwent resection of mesial temporal structures had a recurrence exclusively from the medial temporal lobe. Ramos et al. [14] presented 8 patients with a repeated evaluation after a failed mesial temporal resection and found MRI evidence of residual mesial temporal structures in only one patient. These studies identified dual pathology, relapse from the temporal neocortex and extratemporal foci, as the principal alternative reasons for failure. These causes and others are represented in Figure 1 and discussed in the next sections of the paper.

Some studies have helped understand why some patients need a more extensive resection to achieve seizure freedom. Several authors have pointed out the wide variability in the range of extent of resection and the significant difference between the intended resection and that actually achieved and observed after surgery on brain MRI $[11,15]$. Also Salanova et al. [10] compared the clinical characteristics of TLE patients who failed surgery to those who achieved seizure-free outcomes. The authors found that patients who failed the epilepsy surgery were less likely to have a history of febrile seizures, which is associated with limited damage to vulnerable areas such as the hippocampus, and more likely to have a history of encephalitis or head trauma. These diffuse lesions would result in more extensive epileptogenic areas that are likely to extend to the posterior temporal region. These areas are more difficult to access and less likely to be removed with surgery [16], explaining why these patients had worse seizure outcomes after the first surgery. Following additional resection of the posterior mesial structures and of posterior temporal cortical lesions when present, $57 \%$ of these patients became seizure-free.

The evidence of surgical failure caused by insufficient resection of mesial structures prompted some authors to advocate a more extensive resection during the first surgery [17]. However, results from studies that investigated the relationship between the extent of resection of medial temporal lobe and surgical outcome have been conflicting. Schramm [18] reviewed 12 studies with conclusions on this issue, with 5 authors claiming that a larger extent of resection led to better seizure control, and 7 claiming it did not. A recent randomized controlled study by Schramm et al. $[15,19]$ compared the outcome of two groups of patients who underwent TLE surgery, one with an intended $2.5 \mathrm{~cm}$ resection and the other with a far posterior reaching resection of $3.5 \mathrm{~cm}$. Analysis showed no difference in outcome between both groups. Different resection types (temporal lobectomy or selective amygdalohippocampectomy [SAH]) were used indifferently in both groups, but the authors did not consider that the induced variability in the extent of lateral resection limited their conclusions regarding the extent of mesial resections.

There is still much controversy surrounding the issue of the optimal extent of resection that should be attempted to provide the best outcome in the setting of TLE. Nevertheless, there is sufficient evidence indicating that a subgroup of patients fails TLE surgery because of insufficient resection of mesial temporal structures. These patients are more likely to have history of head trauma or encephalitis. Series of reoperated TLE patients have shown that, in many cases, surgical failure could be transformed into a seizure-free outcome following additional resection of mesial temporal lobe structures. The latest studies investigating the causes of failed TLE surgery report lower rates of patients with residual epileptogenicity in the mesial temporal structures. This could be due to more precise delimitation of the epileptogenic region leading to a more complete surgical resection.

\section{Neocortical Seizure Focus}

Although seizures originate from the mesial portion of the temporal lobe in the majority of TLE cases, approximately 

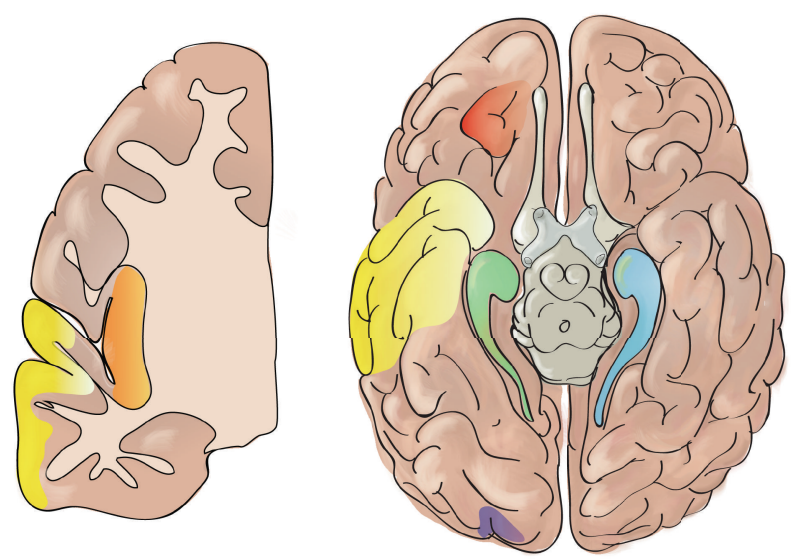

Figure 1: Schematic representation of potential foci responsible for seizure persistence following temporal lobe epilepsy surgery: (a) the ipsilateral mesial temporal structures from incomplete resection (green); (b) the contralateral mesial temporal lobe (blue); (c) the lateral temporal neocortex following selective amygdalohippocampectomy (yellow); (d) the extratemporal lobe epilepsy masquerading as temporal lobe epilepsy such as an orbitofrontal (red), insular (orange), or occipital epileptogenic (purple) zone; (e) the dual pathology such as an unrecognized subtle end-of-sulcus cortical dysplasia in the occipital lobe (purple); (f) a large epileptogenic zone extending to extratemporal structures (temporal epilepsy plus) such as the insula (orange).

$10 \%$ of TLE patients have seizures arising from the lateral temporal lobe [20]. Several studies have aimed to identify clinical, electrographic, and imaging differences between mesial and lateral TLE [20-25], but this distinction can be challenging as some features said to be typical of lateral TLE can also be found in mesial TLE. Although mesial temporal sclerosis is considered a strong indication for mesial TLE, there are some patients with mesial TLE who do not have evidence of MTS, and patients with lateral TLE who can present with this anatomical change [13]. Identification of lateral TLE is even more complicated when the MRI fails to detect a focal lesion on the temporal cortex. Even when the MRI reveals a structural lesion, the site of the lesion is not always indicative of the epileptogenic zone [13, 25, 26]. Holmes et al. [26] reported 20 patients with hippocampal or neocortical lesions on MRI that completely differed in location from the ictal onset. In a study by Kim et al. [25], only $60 \%$ of the structural lesions observed in lateral TLE patients were concordant with the epileptogenic zone. Functional imaging (FDG PET and ictal and interictal SPECT) has been used in an attempt to distinguish the two syndromes, but it may not have sufficient resolution to provide confident discrimination [27].

Identifying the type of TLE and the extent of the epileptogenic zone is important for planning the surgical resection because only a small portion of the temporal neocortex is included in the standard anterior lobectomy. Failure to recognize a neocortical seizure onset would thus often result in surgical failure. Furthermore, Bartolomei et al. [6] quantified the epileptogenicity in the mesial temporal, lateral temporal, and perisylvian regions for 34 patients investigated with stereotactic EEG (SEEG), using a recently proposed measure of epileptogenicity (epileptogenicity index, EI) [28]. In the 5 patients who had "pure" lateral TLE, the epileptogenic zone predominantly involved the superior temporal gyrus and was restricted to this gyrus for 3 of the 5 patients. These findings could support the need of cortical resections extending further than standard anterior temporal lobectomy.

Hennessy et al. [12] reviewed 44 patients who underwent reevaluation to investigate recurrent seizures after temporal resection. Of the 20 patients with mesial temporal sclerosis, 12 had ipsilateral temporal recurrence. Of these patients, only 2 had evidence of seizures arising in the residual hippocampus whereas electroclinical evidence was suggestive of neocortical onset in the other patients. Also, four patients who had electrographic evidence of a neocortical recurrence experienced acoustic auras and speech arrest even before surgery, suggesting that they in fact had unrecognized lateral TLE from the beginning.

Ojemann [13] reported 20 patients with previous temporal resections who continued to have seizures. Of these patients, 19 showed involvement of the mesial temporal structures on the initial evaluation and had resections including parts of the mesial temporal lobe, at least the pes hippocampus. However, postsurgical reevaluation with medial and temporal subdural electrodes found only 4 patients with exclusively mesial temporal focus of recurrence, while 9 of the 20 patients had an exclusively lateral neocortical relapse. Reoperation was only reported for one of the 9 patients with lateral recurrence. The epileptogenic focus was localized to the superior temporal gyrus based on subdural grid and strip electrodes and the patient achieved seizure freedom after additional lateral temporal cortex resection.

Similarly, Ramos et al. [14] reported 5 patients with MTS and ipsilateral temporal seizure recurrence after selective anterior mesial temporal resection (removal of the hippocampus and surrounding parahippocampus to the level of the superior colliculi). One of these patients had an intentionally incomplete resection of mesial structures and benefited from further resection of these structures. All the 4 other patients were thought after the first surgery to have 
neocortical seizure onset and consequently had extended neocortical resection along the previous resection cavity in the temporal lobe. Two of the four patients (50\%) achieved an Engel class I or II outcome.

All these studies stress the importance of identifying an epileptogenic temporal neocortex during the presurgical evaluation to prevent surgical failure in some patients. Several authors have pointed out that some patients show variable implications of both the mesial and lateral parts of the temporal lobe in seizure onset $[13,29]$. This raises the complicated issue of dual pathology, where both parts of the temporal lobe are thought to be epileptogenic.

\section{Dual Pathology}

Dual pathology refers to the coexistence of mesial temporal sclerosis and extrahippocampal lesion. The lesion is most commonly temporal but can also be extratemporal. Neuroimaging studies report dual pathological conditions in approximately $15 \%$ of patients with TLE evaluated for surgery [30-32]. When the diagnosis is based on pathological examination, dual pathology is found in $30 \%$ of surgical specimens of patients operated for TLE [30, 33]. The most common extrahippocampal lesions are developmental abnormalities such as cortical dysplasia. In a series of 38 patients with dual pathology [31], cortical dysplasia accounted for $47 \%$ of the lesions associated with mesial temporal sclerosis, but rates as high as $73 \%$ have been reported elsewhere [32]. Low-grade tumors are the second most common finding in dual pathology (26\%), followed by cerebral contusions and infarcts $(21 \%)$ and vascular malformations (13\%) [31].

The pathogenesis of dual pathology is not well understood yet, and multiple hypotheses have been formulated. One proposed explanation is that the lesion and the mesial temporal sclerosis are both caused by a common pathological mechanism. According to this hypothesis, a genetic or environmental insult disturbing normal embryogenesis and neuronal migration could result in an abnormal and atrophic hippocampus, in addition to a structural lesion such as cortical dysplasia [30, 34, 35]. A common pathological event in the perinatal period, such as perinatal ischemia, could also result in dual pathology [36].

An alternative explanation for the development of dual pathology holds that the pathological mechanism causing cortical dysplasia could also affect the hippocampus by increasing its susceptibility to febrile seizures, thus favouring the development of MTS $[34,35]$. Cortical dysplasia has been shown to increase the frequency of hyperthermia-induced seizures in rats [37]. However, a study by Raymond et al. [35] has provided evidence against this theory, as only 2 of 15 patients with hippocampal sclerosis and cortical dysplasia had a history of febrile seizures.

The lesions associated with dual pathology are most commonly congenital or acquired early in life and have an epileptogenic potential. A third proposed hypothesis for dual pathology is that repetitive seizures originating from these early epileptogenic lesions could result in neuronal loss in the vulnerable hippocampus and lead to mesial temporal sclerosis $[35,38,39]$. These early seizures could also cause the generation of a secondary epileptogenic focus in the mesial temporal region, as the younger brain is thought to be more susceptible to secondary epileptogenesis $[32,40]$.

As a high proportion of children operated for intractable TLE has pathologically confirmed dual pathology (24$100 \%)$, it is hypothesized that dual pathology results in intractable epilepsy earlier in life, prompting earlier referral to surgery [41]. Several studies have stressed that optimal surgical strategy requires resection of both the lesion and the sclerotic mesial temporal structures [31, 32]. When both are removed, the seizure outcome is similar to that in patients with mesial temporal sclerosis alone $[4,32$, 41, 42]. A multicentre study by $\mathrm{Li}$ et al. [31] reported 41 surgical interventions in patients with dual pathology. Resection of the lesion and the mesial temporal structures resulted in seizure freedom in $11(73 \%)$ of 15 patients, while lesionectomy or resection of mesial temporal structures alone resulted in seizure freedom for only $2(12.5 \%)$ of 16 patients and $2(20 \%)$ of 10 patients, respectively. Another study by $\mathrm{Li}$ et al. [43] reported the surgical outcome of 13 patients with dual pathology. The three patients who underwent resection of both the lesion and the sclerotic mesial structures all achieved a seizure-free outcome. The ten others had resection of only one of the two pathologies, with only $2(20 \%)$ becoming seizure-free.

In a prospective study by Cascino et al. [42], 15 patients with extrahippocampal lesions identified on MRI underwent stereotactic lesionectomy. Three of the 15 patients presented MRI evidence of hippocampal atrophy in addition to the lesion, with all three having an unsatisfactory seizure outcome (Engel class IV) after the lesionectomy. On the other hand, 7 of the 12 patients without hippocampal atrophy achieved seizure freedom, and 2 others experienced $\geq 90 \%$ reduction in seizures. The three patients with hippocampal atrophy underwent a second operation (lobectomy with amygdalohippocampectomy) that resulted in a seizure-free outcome in all of them. Hence, failure to identify and resect the two coexistent pathologies could be an important explanation for failure of TLE surgery.

One of the challenges in the treatment of patients with dual pathology is initial identification of the lesion, as mild changes of dysplasia may not be seen with current neuroimaging techniques $[11,12,44]$. The study by $\mathrm{Li}$ and colleagues [31] found that even subtle signs of MTS had a negative impact on seizure outcome if mesial temporal structures were not removed in addition to the lesion. These signs of minimal MTS can be missed on routine presurgical MRI, leading to the authors' conclusion that quantitative MRI may be of value in identifying subtle hippocampal atrophy in patients with greater risk of presenting dual pathology, namely, those with identified early developmental lesions [30, 31]. In fact, some authors have reported that as many as $90 \%$ of patients with temporal lobe developmental abnormalities have concurrent atrophy of the mesial structures $[45,46]$. As mild cortical dysplasia can also be missed by the current techniques of neuroimaging, the role of dual pathology in surgical failures of TLE surgery is thought to 
be even more important than reported, with some authors claiming that it may even be the principal cause of failure following temporal resection [41].

\section{Contralateral Relapse}

Seizure relapse from the temporal lobe contralateral to the side of surgery is an intuitive and important cause of failure in the surgical treatment of TLE, estimated to represent 12 $30 \%$ of patients who fail surgery $[10,12-14,47]$.

Hennessy et al. [12] identified contralateral temporal relapse in $20 \%$ of all TLE surgical failures and in as many as $30 \%$ when considering only patients with MTS. Of 8 patients with failed TLE surgery, Ramos et al. [14] reported 3 patients (38\%) who had EEG evidence of contralateral relapse. Only one patient had bilateral interictal spikes but with $>80 \%$ predominance to the surgical side. Jehi et al. [47] reported that 13 of 44 patients (30\%) who failed surgery had a seizure focus in the contralateral temporal lobe. Salanova et al. [10] reviewed 41 patients with failed TLE surgery and found 5 patients (12\%) with contralateral seizure onset.

These rates of contralateral relapses are not surprising, as neuropathological studies have frequently described bilateral involvement of the temporal lobes in mesial temporal sclerosis. Autopsy studies of patients with temporal lobe epilepsy reported rates of bilateral MTS ranging from 10 to $80 \%$ [48-50]. In addition, secondary epileptogenesis has been proposed as a mechanism for the development of a contralateral focus in patients with temporal lobe epilepsy [40, 48, 51]. Morrell and detoledo-Morrell [40] analyzed 68 patients with tumors presenting with epilepsy, 26 (38\%) of whom showed evidence of contralateral independent foci. After surgical resection of the tumor, 8 of the 26 patients continued to have seizures arising from the side contralateral to the removed tumor. The authors considered these 8 patients to be in the independent stage of secondary epileptogenesis, the stage at which the process is irreversible even after removal of the primary focus. Similar results were reported by Gilmore et al. [52], who found 7 cases of secondary focus in a series of 22 patients with temporal lobe tumor.

The outcome for patients with confirmed bitemporal independent ictal activity is less favorable than for patients with unilateral seizure onset [11, 47, 53-55]. Jehi et al. [47] evaluated the outcome of TLE surgery in 371 patients and reported that bilateral MR imaging abnormalities reduced the probability of seizure freedom at 2 years from $78 \%$ to 58\%. Also, Abosch et al. [11] compared 27 TLE patients who failed surgery (Engel class III and IV) to 27 who achieved seizure freedom, and they identified evidence of bilateral temporal onset as a predictor of poor postoperative seizure control. Nonetheless, recent studies have reported good results from surgery for bitemporal lobe epilepsy, suggesting that patients suffering from bitemporal epilepsy should still be evaluated for surgery [53, 55-58]. Boling et al. [55] reported 11 cases of surgery for independent bitemporal lobe epilepsy, 7 of whom achieved a satisfactory outcome (6 in Engel class I, 1 in Engel class II).
Bilateral temporal lobe epilepsy is a complicated issue. In addition to having a poorer outcome than unilateral TLE $[5,59]$, there is still some controversy surrounding the clinical and electrographic evidence that should be used to choose the surgical side $[55,58]$. Several studies have achieved good surgical outcomes in patients with bitemporal epilepsy when a sufficient proportion of seizures arises from one temporal lobe. However, the degree of lateralization required is still debated. So et al. [60] reported that 8 (53\%) of 15 patients with $\geq 80 \%$ of seizures originating from the resected temporal lobe achieved seizure freedom, compared to none of the 6 patients with $<80 \%$ of lateralization. In a study by Holmes et al. [58], 5 of 7 bitemporal patients had $\geq 80 \%$ of seizures beginning on one side, and all five were either seizure-free or had $>75 \%$ reduction in seizure frequency after the operation. The 2 other patients had $<80 \%$ seizure arising from the resected lobe and none experienced a significant reduction in seizure frequency ( $<75 \%$ reduction). Conversely, Hirsch et al. [56] reported excellent surgical outcome in 11 patients (10 seizure-free and one $\geq 75 \%$ reduction) with only $\geq 55 \%$ of seizures arising from the resected temporal lobe, albeit with complimentary tests (e.g., amobarbital test and imaging studies) strongly favoring the resected side.

Repeated resection on the contralateral side is not an option for these patients. Nevertheless, the reported rates of seizure freedom following unilateral resective surgery for medically intractable patients is still much higher than what can be achieved by pharmacotherapy alone, meaning that surgery can still play an important role in the management of these patients [55].

\section{Extratemporal and Temporal Plus}

Recurrence from an extratemporal focus after a temporal lobe resection in patients with TLE is a commonly reported cause of surgical failure, accounting for $14-20 \%$ of cases in studies investigating the mechanisms of failure of TLE surgery $[12,13,47]$. It is well recognized that some cases of extratemporal epilepsy syndromes can be mistaken for TLE syndrome on the basis of both clinical manifestations and electrographic studies, particularly when only scalp videoEEG monitoring is performed [54]. Lee et al. reported 33 consecutive nonlesional patients diagnosed with TLE, all with evidence of an exclusively temporal ictal onset zone based on scalp video-EEG monitoring. Using intracranial grid and strip recording, $11(33 \%)$ of these 33 patients were actually found to have extratemporal seizure onset (3 frontal, 5 parietal, 1 occipital, and 2 temporoparietal). Distinguishing between temporal and extratemporal epilepsy is also complicated by the fact that extratemporal seizures can mimic the clinical features of TLE. This has been reported in the setting of insular [61,62], orbitofrontal [63], and cingulate gyrus [64] epilepsy syndromes. Also, some seizures arising in extratemporal areas can remain nearly asymptomatic until they spread to the temporal lobe, where they can trigger TLE syndrome and be recorded. These cases 
are designated as "pseudotemporal epilepsy" [27] and have been described in occipital [65] and parietal [66] epilepsy.

Alternately, it has been suggested that some patients with surgically refractory TLE could actually suffer from a more complex epileptogenic network that can encompass the temporal lobe and brain regions to which it is closely related, such as the orbitofrontal cortex, the insula, the frontal and parietal opercula, and the temporoparietooccipital junction [67]. Ryvlin and Kahane recently introduced the term temporal plus epilepsy (TPE) to designate this form of multilobar epilepsy. A study by the same group identified 281 patients who underwent temporal lobe surgery and had preoperative SEEG and found that as many as $60(21 \%)$ patients had an epileptogenic zone extending beyond the temporal lobe (temporoparietooccipital, orbitofrontal, or perisylvian area including the insula) [68]. Distinguishing between TLE and TPE on the basis of noninvasive studies can be very difficult [69], meaning that patients with multilobar involvement can be easily misdiagnosed as having a typical temporal lobe epilepsy syndrome. This would lead to incomplete resection of the epileptogenic region and surgical failure. Ryvlin et al. [70] showed that only $9 \%$ of TPE patients who underwent a standard anterior lobectomy achieved an Engel class I outcome, compared to $74 \%$ in whom the surgical resection was tailored to fully include the epileptogenic cortex. Failure to recognize TPE could thus explain part of the surgical failures in TLE surgery.

\section{The Role of the Insula in TLE Surgery Failure}

The role of the insula in explaining failures of TLE surgery has recently gained a lot of attention $[61,62,67,71-76]$. In the early 1950s, Penfield had already observed that seizures arising from the insula could mimic temporal lobe seizures, so that both could be confused [77]. Insular seizures can manifest at onset as early visceral symptoms or dysphasia with alteration of consciousness, suggesting temporal lobe seizures $[61,74,76]$. Insular epilepsy can also accompany temporal lobe epilepsy as part of a multilobar epileptogenic zone (TPE) [61, 73]. In such cases, temporal lobectomy alone has been shown to be inefficient. In a series of 21 patients with atypical TLE reported by Isnard et al. [61], two patients showed SEEG evidence of spontaneous insular seizures, and both achieved a poor outcome (Engel class IV) following temporal lobectomy only.

The contribution of noninvasive investigations in the detection of insular epilepsy masquerading as or concomitant to TLE is limited. The most useful technique is MRI, as visualization of an insular lesion strongly supports the likelihood of insular involvement [75]. Unfortunately, insular epilepsy is commonly nonlesional [78]. Scalp EEG is of limited utility as it is unable to distinguish insular seizures from perisylvian, parietal, frontal, or temporal lobe seizures $[69,73,76]$. SPECT and PET scans may reveal changes in the insular lobe, but their specificity is low [75]. There is some promising evidence that magnetoencephalography (MEG) can be useful in localizing insular epileptogenic foci, even when other noninvasive tests have failed [79], but further studies are needed to assess its true clinical utility. In the absence of a visualized lesion on MR imaging, intracranial recording with insular sampling is necessary. Several implantation methods have been proposed, providing variable coverage of the insula and the perisylvian cortex $[61,76,80,81]$.

Clinical features can help guide the selection of patients who should undergo invasive investigation. Particularly, patients with temporal lobe epilepsy symptoms who also show "atypical" features such as occurrence at onset of somatosensory (e.g., laryngeal discomfort, throat constriction, limb paresthesias) or motor symptoms (e.g., arm elevation, trashing, or pedaling) should undergo an intracranial study with proper sampling of the insula [76].

In an attempt to improve the outcome of temporal lobe epilepsy surgery, Penfield was the first to perform insular resection, when residual epileptiform activity in the insula was recorded after removal of the temporal lobe. However, this approach was abandoned after Silfvenius et al. [82] reported that this method did not benefit seizure control while significantly increased morbidity from 3 to $21 \%$ (mainly hemiparesis) [82]. Since then and until recently, the possible role of the insula in temporal lobe epilepsy was disregarded. It is of interest that patients in the same study by Silfvenius et al. who underwent reoperation and had an insulectomy had a rate of unsatisfactory outcome of only $46 \%$ compared to $83 \%$ in patients who did not have insulectomy, suggesting that the insula could actually play a part in the surgical failure of some patients with TLE.

Several publications [83-88] have recently shown that insular epilepsy surgery can be both safe and beneficial with modern neurosurgical techniques. Our group recently reported a series of 7 insulectomies for refractory epilepsy [85], with 6 patients achieving an Engel class IA outcome, and the seventh also becoming seizure-free following insular Gamma Knife surgery. The postoperative deficits consisted in transient hemiparesis in 57\% (4/7) of the patients, with no permanent deficit encountered.

This decreased morbidity of insular surgery makes it even more important to understand the role of the insula in failure of epilepsy surgery, as resection of this lobe could transform a poor outcome into a seizure-free outcome for an undetermined proportion of TLE patients (Figure 2). Hopefully, further clinical observations and use of modern noninvasive neuroimaging techniques (magnetoencephalography, combined EEG-fMRI, magnetic resonance spectroscopy, quantitative MRI, etc.) should allow us to better identify the subset of patients who would most benefit from insular intracerebral electrodes $[75,80,81]$ and/or insular resection.

\section{Conclusion}

Patients with TLE who have persistence or recurrence of seizures following resective surgery most likely follow one of the five relapse patterns identified and explained in this review. While insufficient resection of mesial temporal structures was frequently cited as the most common reason 

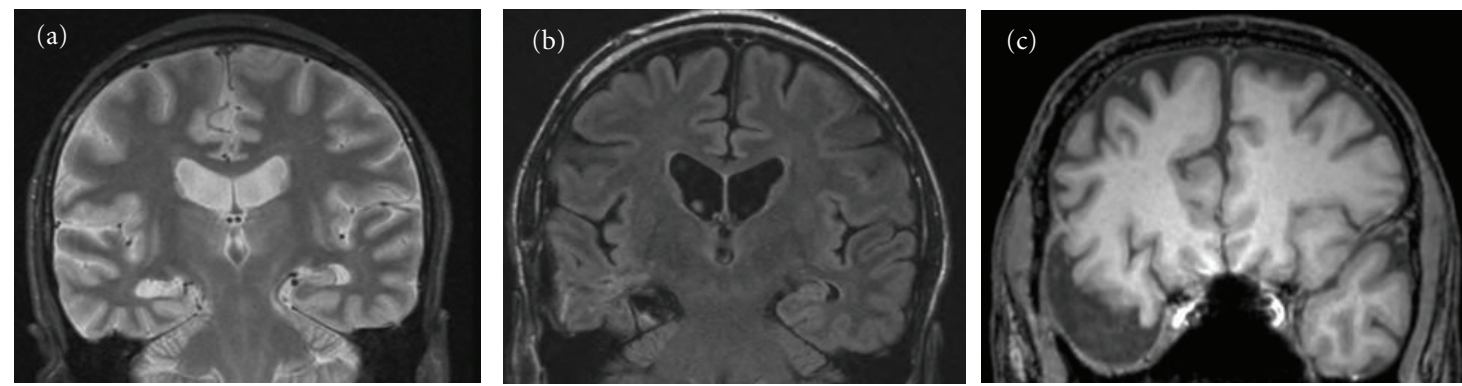

(d) Fp1-F7
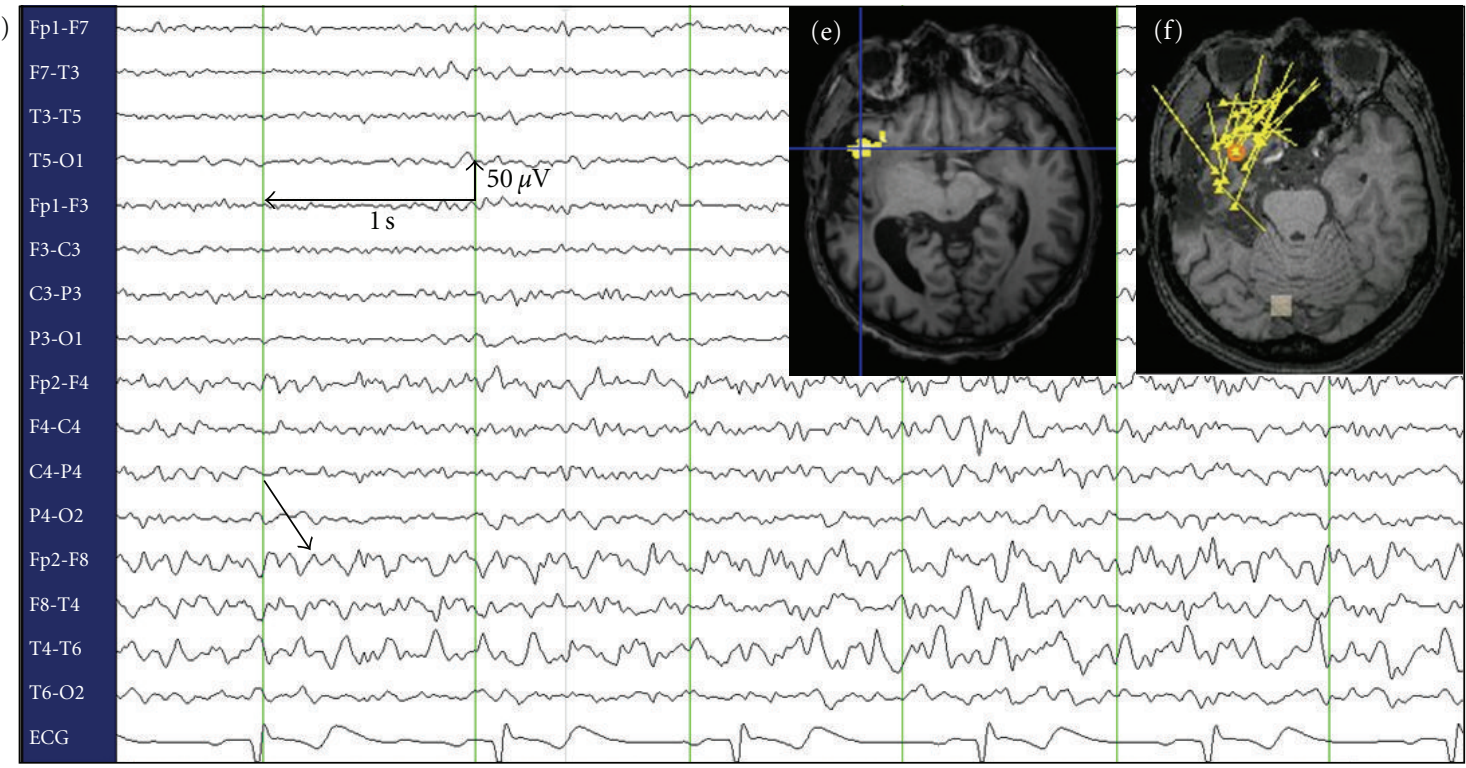

(g)

(1)

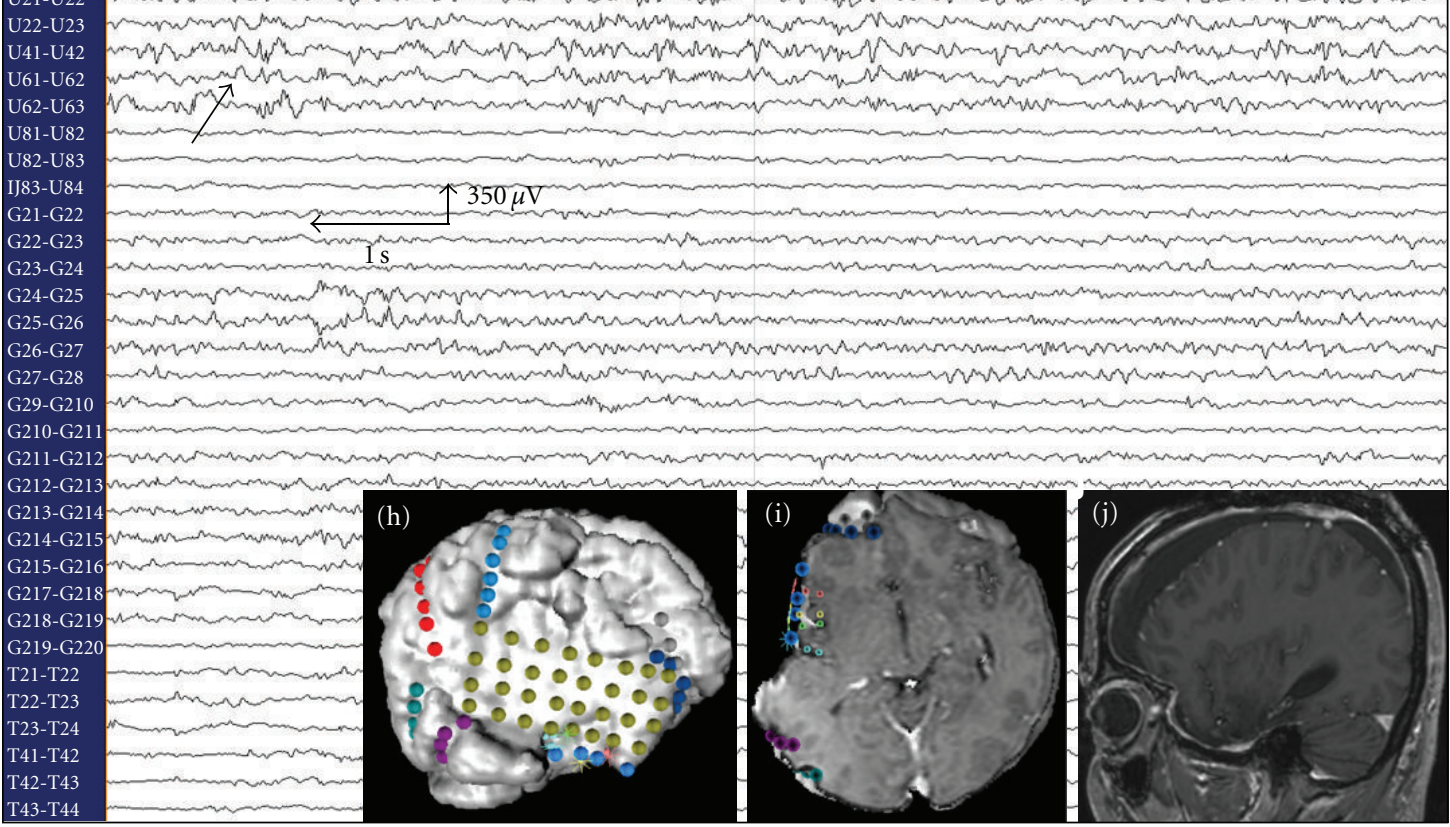

FIgURE 2: Case of insular epilepsy masquerading as TLE operated at our institution. (a) MRI showing right hippocampal sclerosis in a 52-year-old patient with medically refractory epilepsy since age 20. (b) Presurgical evaluation led to a selective amygdalohippocampectomy that failed to control seizures. (c) Temporal lobectomy was subsequently performed, but the patient still continued to have seizures. (d): Postlobectomy scalp EEG continued to show ipsilateral temporal epileptiform activity. (e) and (f) fMRI-EEG and MEEG, respectively, localized the epileptogenic zone to the ipsilateral right insular cortex. (g) Intracranial EEG identified an epileptogenic focus in the anterior insular cortex. (h) and (i) Implantation of the intracranial electrodes. (j) MRI following partial anteroinferior insulectomy. The patient has been seizure-free since the operation (followup: 6 months). 
for seizure relapse a decade ago, recent studies have stressed the importance of dual pathology and extratemporal epileptogenic zones in surgical failure. These causes are believed to involve epileptogenic networks instead of a delimited focus. Better understanding of the causes behind these failures is necessary to improve the surgical outcome of patients with medically intractable epilepsy.

\section{References}

[1] J. W. Sander, Y. M. Hart, A. L. Johnson, and S. D. Shorvon, "National general practice study of epilepsy: newly diagnosed epileptic seizures in a general population," The Lancet, vol. 336, no. 8726, pp. 1267-1271, 1990.

[2] P. Kwan and M. J. Brodie, "Early identification of refractory epilepsy," The New England Journal of Medicine, vol. 342, no. 5, pp. 314-319, 2000.

[3] S. Wiebe, W. T. Blume, J. P. Girvin, and M. Eliasziw, "A randomized, controlled trial of surgery for temporal-lobe epilepsy," The New England Journal of Medicine, vol. 345, no. 5, pp. 311-318, 2001.

[4] A. M. McIntosh, R. M. Kalnins, L. A. Mitchell, G. C. A. Fabinyi, R. S. Briellmann, and S. F. Berkovic, "Temporal lobectomy: long-term seizure outcome, late recurrence and risks for seizure recurrence," Brain, vol. 127, no. 9, pp. 2018 2030, 2004.

[5] L. E. Jeha, I. M. Najm, W. E. Bingaman et al., "Predictors of outcome after temporal lobectomy for the treatment of intractable epilepsy," Neurology, vol. 66, no. 12, pp. 1938-1940, 2006.

[6] F. Bartolomei, D. Cosandier-Rimele, A. McGonigal et al., "From mesial temporal lobe to temporoperisylvian seizures: a quantified study of temporal lobe seizure networks," Epilepsia, vol. 51, no. 10, pp. 2147-2158, 2010.

[7] A. R. Wyler, B. P. Hermann, and E. T. Richey, "Results of reoperation for failed epilepsy surgery," Journal of Neurosurgery, vol. 71, no. 6, pp. 815-819, 1989.

[8] I. A. Awad, M. H. Nayel, and H. Luders, "Second operation after the failure of previous resection for epilepsy," Neurosurgery, vol. 28, no. 4, pp. 510-518, 1991.

[9] I. M. Germano, N. Poulin, and A. Olivier, "Reoperation for recurrent temporal lobe epilepsy," Journal of Neurosurgery, vol. 81, no. 1, pp. 31-36, 1994.

[10] V. Salanova, O. Markand, and R. Worth, "Temporal lobe epilepsy: analysis of failures and the role of reoperation," Acta Neurologica Scandinavica, vol. 111, no. 2, pp. 126-133, 2005.

[11] A. Abosch, N. Bernasconi, W. Boling et al., "Factors predictive of suboptimal seizure control following selective amygdalohippocampectomy," Journal of Neurosurgery, vol. 97, no. 5, pp. 1142-1151, 2002.

[12] M. J. Hennessy, R. D. Elwes, C. D. Binnie, and C. E. Polkey, "Failed surgery for epilepsy: A study of persistence and recurrence of seizures following temporal resection," Brain, vol. 123, no. 12, pp. 2445-2466, 2000.

[13] G. A. Ojemann, "Interplay between "neocortical" and "limbic" temporal lobe epilepsy," Advances in Neurology, vol. 84, pp. 615-619, 2000.

[14] E. Ramos, S. Benbadis, and F. L. Vale, "Failure of temporal lobe resection for epilepsy in patients with mesial temporal sclerosis: results and treatment options: clinical article," Journal of Neurosurgery, vol. 110, no. 6, pp. 1127-1134, 2009.
[15] J. Schramm, T. N. Lehmann, J. Zentner et al., "Randomized controlled trial of $2.5-\mathrm{cm}$ versus $3.5-\mathrm{cm}$ mesial temporal resection in temporal lobe epilepsy-Part 1: intent-to-treat analysis," Acta Neurochir, vol. 153, no. 2, pp. 209-219, 2011.

[16] D. D. Spencer, S. S. Spencer, R. H. Mattson, P. D. Williamson, and R. A. Novelly, "Access to the posterior medial temporal lobe structures in the surgical treatment of temporal lobe epilepsy," Neurosurgery, vol. 15, no. 5, pp. 667-671, 1984.

[17] A. R. Wyler, B. P. Hermann, and G. Somes, "Extent of medial temporal resection on outcome from anterior temporal lobectomy: a randomized prospective study," Neurosurgery, vol. 37, no. 5, pp. 982-991, 1995.

[18] J. Schramm, "Temporal lobe epilepsy surgery and the quest for optimal extent of resection: a review," Epilepsia, vol. 49, no. 8, pp. 1296-1307, 2008.

[19] J. Schramm, T.N. Lehmann, J. Zentner et al., "Randomized controlled trial of $2.5-\mathrm{cm}$ versus $3.5-\mathrm{cm}$ mesial temporal resection-part 2: volumetric resection extent and subgroup analyses," Acta Neurochir, vol. 153, no. 2, pp. 221-228, 2011.

[20] J. Schramm, T. Kral, T. Grunwald, and I. Blümcke, "Surgical treatment for neocortical temporal lobe epilepsy: clinical and surgical aspects and seizure outcome," Journal of Neurosurgery, vol. 94, no. 1, pp. 33-42, 2001.

[21] R. S. Burgerman, M. R. Sperling, J. A. French, A. J. Saykin, and M. J. O'Connor, "Comparison of mesial versus neocortical onset temporal lobe seizures: neurodiagnostic findings and surgical outcome," Epilepsia, vol. 36, no. 7, pp. 662-670, 1995.

[22] S. V. Pacia, O. Devinsky, K. Perrine et al., "Clinical features of neocortical temporal lobe epilepsy," Annals of Neurology, vol. 40, no. 5, pp. 724-730, 1996.

[23] A. Gil-Nagel and M. W. Risinger, "Ictal semiology in hippocampal versus extrahippocampal temporal lobe epilepsy," Brain, vol. 120, no. 1, pp. 183-192, 1997.

[24] N. Foldvary, N. Lee, G. Thwaites et al., "Clinical and electrographic manifestations of lesional neocortical temporal lobe epilepsy," Neurology, vol. 49, no. 3, pp. 757-763, 1997.

[25] Y. K. Kim, D. S. Lee, S. K. Lee et al., "Differential features of metabolic abnormalities between medial and lateral temporal lobe epilepsy: quantitative analysis of (18)F-FDG PET using SPM," Journal of Nuclear Medicine, vol. 44, no. 7, pp. 10061012, 2003.

[26] M. D. Holmes, A. J. Wilensky, G. A. Ojemann, and L. M. Ojemann, "Hippocampal or neocortical lesions on magnetic resonance imaging do not necessarily indicate site of ictal onsets in partial epilepsy," Annals of Neurology, vol. 45, no. 4, pp. 461-465, 1999.

[27] F. Andermann, "Pseudotemporal vs neocortical temporal epilepsy: things aren't always where they seem to be," Neurology, vol. 61, no. 6, pp. 732-733, 2003.

[28] F. Bartolomei, P. Chauvel, and F. Wendling, "Epileptogenicity of brain structures in human temporal lobe epilepsy: a quantified study from intracerebral EEG," Brain, vol. 131, no. 7, pp. 1818-1830, 2008.

[29] L. Maillard, J. P. Vignal, M. Gavaret et al., "Semiologic and electrophysiologic correlations in temporal lobe seizure subtypes," Epilepsia, vol. 45, no. 12, pp. 1590-1599, 2004.

[30] F. Cendes, M. J. Cook, C. Watson et al., "Frequency and characteristics of dual pathology in patients with lesional epilepsy," Neurology, vol. 45, no. 11, pp. 2058-2064, 1995.

[31] L. M. Li, F. Cendes, F. Andermann et al., "Surgical outcome in patients with epilepsy and dual pathology," Brain, vol. 122, no. 5, pp. 799-805, 1999. 
[32] V. Salanova, O. Markand, and R. Worth, "Temporal lobe epilepsy: analysis of patients with dual pathology," Acta Neurologica Scandinavica, vol. 109, no. 2, pp. 126-131, 2004.

[33] M. F. Levesque, N. Nakasato, H. V. Vinters, and T. L. Babb, "Surgical treatment of limbic epilepsy associated with extrahippocampal lesions: the problem of dual pathology," Journal of Neurosurgery, vol. 75, no. 3, pp. 364-370, 1991.

[34] C. Bocti, Y. Robitaille, P. Diadori et al., "The pathological basis of temporal lobe epilepsy in childhood," Neurology, vol. 60, no. 2, pp. 191-195, 2003.

[35] A. A. Raymond, D. R. Fish, J. M. Stevens, M. J. Cook, S. M. Sisodiya, and S. D. Shorvon, "Association of hippocampal sclerosis with cortical dysgenesis in patients with epilepsy," Neurology, vol. 44, no. 10, pp. 1841-1845, 1994.

[36] A. Rougier, "Dual pathology," Neurochirurgie, vol. 54, no. 3, pp. 382-387, 2008.

[37] I. M. Germano, Y. F. Zhang, E. F. Sperber, and S. L. Moshé, "Neuronal migration disorders increase susceptibility to hyperthermia- induced seizures in developing rats," Epilepsia, vol. 37, no. 9, pp. 902-910, 1996.

[38] R. S. Fisher and D. Blum, "Epilepsy surgery where there is dual pathology," The Lancet, vol. 354, no. 9175, pp. 267-268, 1999.

[39] S. H. Eriksson, C. Nordborg, B. Rydenhag, and K. Malmgren, "Parenchymal lesions in pharmacoresistant temporal lobe epilepsy: dual and multiple pathology," Acta Neurologica Scandinavica, vol. 112, no. 3, pp. 151-156, 2005.

[40] F. Morrell and L. deToledo-Morrell, "From mirror focus to secondary epileptogenesis in man: an historical review," Advances in neurology, vol. 81, pp. 11-23, 1999.

[41] S. Spencer and L. Huh, "Outcomes of epilepsy surgery in adults and children," The Lancet Neurology, vol. 7, no. 6, pp. 525-537, 2008.

[42] G. D. Cascino, C. R. Jack Jr., J. E. Parisi et al., "Operative strategy in patients with MRI-identified dual pathology and temporal lobe epilepsy," Epilepsy Research, vol. 14, no. 2, pp. 175-182, 1993.

[43] L. M. Li, F. Cendes, C. Watson et al., "Surgical treatment of patients with single and dual pathology: relevance of lesion and of hippocampal atrophy to seizure outcome," Neurology, vol. 48, no. 2, pp. 437-444, 1997.

[44] R. Kuzniecky, J. H. Garcia, E. Faught, and R. B. Morawetz, "Cortical dysplasia in temporal lobe epilepsy: magnetic resonance imaging correlations," Annals of Neurology, vol. 29, no. 3, pp. 293-298, 1991.

[45] S. S. Ho, R. I. Kuzniecky, F. Gilliam, E. Faught, and R. Morawetz, "Temporal lobe developmental malformations and epilepsy: dual pathology and bilateral hippocampal abnormalities," Neurology, vol. 50, no. 3, pp. 748-754, 1998.

[46] T. H. Schwartz and D. D. Spencer, "Strategies for reoperation after comprehensive epilepsy surgery," Journal of Neurosurgery, vol. 95, no. 4, pp. 615-623, 2001.

[47] L. E. Jehi, D. C. Silveira, W. Bingaman, and I. Najm, “Temporal lobe epilepsy surgery failures: predictors of seizure recurrence, yield of reevaluation, and outcome following reoperation," Journal of Neurosurgery, vol. 113, no. 6, pp. 1186-1194, 2010.

[48] H. J. Meencke, G. Veith, and S. Lund, "Bilateral hippocampal sclerosis and secondary epileptogenesis," Epilepsy Research. Supplement, vol. 12, pp. 335-342, 1996.

[49] S. S. Spencer, "Substrates of localization-related epilepsies: biologic implications of localizing findings in humans," Epilepsia, vol. 39, no. 2, pp. 114-123, 1998.

[50] G. W. Mathern, T. L. Babb, and D. L. Armstrong, "Hippocampal sclerosis," in Epilepsy: A Comprehensive Textbook,
J. Engel Jr. and T. A. Pedley, Eds., vol. 1, pp. 133-155, Lippincott-Raven, Philadelphia, Pa, USA, 1997.

[51] F. Morrell, "Secondary epileptogenesis in man," Archives of Neurology, vol. 42, no. 4, pp. 318-335, 1985.

[52] R. Gilmore, H. Morris III, P. C. van Ness, W. Gilmore-Pollak, and M. Estes, "Mirror focus: function of seizure frequency and influence on outcome after surgery," Epilepsia, vol. 35, no. 2, pp. 258-263, 1994.

[53] J. I. Sirven, B. L. Malamut, J. D. Liporace, M. J. O’Connor, and M. R. Sperling, "Outcome after temporal lobectomy in bilateral temporal lobe epilepsy," Annals of Neurology, vol. 42, no. 6, pp. 873-878, 1997.

[54] K. H. Lee, Y. D. Park, D. W. King et al., "Prognostic implication of contralateral secondary electrographic seizures in temporal lobe epilepsy," Epilepsia, vol. 41, no. 11, pp. 1444-1449, 2000.

[55] W. Boling, Y. Aghakhani, F. Andermann, V. Sziklas, and A. Olivier, "Surgical treatment of independent bitemporal lobe epilepsy defined by invasive recordings," Journal of Neurology, Neurosurgery \& Psychiatry, vol. 80, no. 5, pp. 533-538, 2009.

[56] L. J. Hirsch, S. S. Spencer, D. D. Spencer, P. D. Williamson, and R. H. Mattson, "Temporal lobectomy in patients with bitemporal epilepsy defined by depth electroencephalography," Annals of Neurology, vol. 30, no. 3, pp. 347-356, 1991.

[57] M. D. Holmes, C. B. Dodrill, G. A. Ojemann, A. J. Wilensky, and L. M. Ojemann, "Outcome following surgery in patients with bitemporal interictal epileptiform patterns," Neurology, vol. 48, no. 4, pp. 1037-1040, 1997.

[58] M. D. Holmes, A. N. Miles, C. B. Dodrill, G. A. Ojemann, and A. J. Wilensky, "Identifying potential surgical candidates in patients with evidence of bitemporal epilepsy," Epilepsia, vol. 44, no. 8, pp. 1075-1079, 2003.

[59] J. Janszky, H. W. Pannek, I. Janszky et al., "Failed surgery for temporal lobe epilepsy: predictors of long-term seizure-free course," Epilepsy Research, vol. 64, no. 1-2, pp. 35-44, 2005.

[60] N. So, A. Olivier, F. Andermann, P. Gloor, and L. F. Quesney, "Results of surgical treatment in patients with bitemporal epileptiform abnormalities," Annals of Neurology, vol. 25, no. 5, pp. 432-439, 1989.

[61] J. Isnard, M. Guenot, K. Ostrowsky, M. Sindou, and F. Mauguière, "The role of the insular cortex in temporal lobe epilepsy," Annals of Neurology, vol. 48, no. 4, pp. 614-623, 2000.

[62] P. Ryvlin, "Beyond pharmacotherapy: surgical management," Epilepsia, vol. 44, supplement 5, pp. 23-28, 2003.

[63] B. Shihabuddin, B. Abou-Khalil, D. Delbeke, and T. Fakhoury, "Orbito-frontal epilepsy masquerading as temporal lobe epilepsy-a case report," Seizure, vol. 10, no. 2, pp. 134-138, 2001.

[64] O. Devinsky, M. J. Morrell, and B. A. Vogt, "Contributions of anterior cingulate cortex to behaviour," Brain, vol. 118, no. 1, pp. 279-306, 1995.

[65] A. Palmini, F. Andermann, F. Dubeau et al., "Occipitotemporal epilepsies: evaluation of selected patients requiring depth electrodes studies and rationale for surgical approaches," Epilepsia, vol. 34, no. 1, pp. 84-96, 1993.

[66] P. D. Williamson, P. A. Boon, V. M. Thadani et al., "Parietal lobe epilepsy: diagnostic considerations and results of surgery," Annals of Neurology, vol. 31, no. 2, pp. 193-201, 1992.

[67] P. Ryvlin and P. Kahane, "The hidden causes of surgeryresistant temporal lobe epilepsy: extratemporal or temporal plus?" Current Opinion in Neurology, vol. 18, no. 2, pp. 125127,2005 
[68] P. Kahane, J. Isnard, D. Hoffmann et al., "Temporal plus epilepsies I: prevalence in epilepsy surgery patients," Epilepsia, vol. 42, supplement 7, article 192, 2001.

[69] C. Barba, G. Barbati, L. Minotti, D. Hoffmann, and P. Kahane, "Ictal clinical and scalp-EEG findings differentiating temporal lobe epilepsies from temporal "plus" epilepsies," Brain, vol. 130, no. 7, pp. 1957-1967, 2007.

[70] P. Ryvlin, P. Kahane, and J. Isnard, "Temporal plus epilepsies II: surgical results," Epilepsia, vol. 42, supplement 7, article 198, 2001.

[71] J. Isnard, M. Guénot, M. Sindou, and F. Mauguière, "Clinical manifestations of insular lobe seizures: a stereo- electroencephalographic study," Epilepsia, vol. 45, no. 9, pp. 1079-1090, 2004.

[72] P. Ryvlin, "Avoid falling into the depths of the insular trap," Epileptic Disorders, vol. 8, supplement 2, pp. S37-S56, 2006.

[73] D. K. Nguyen, D. B. Nguyen, R. Malak, and A. Bouthillier, "Insular cortex epilepsy: an overview," Canadian Journal of Neurological Sciences, vol. 36, supplement 2, pp. S58-S62, 2009.

[74] D. K. Nguyen, D. B. Nguyen, R. Malak et al., "Revisiting the role of the insula in refractory partial epilepsy," Epilepsia, vol. 50, no. 3, pp. 510-520, 2009.

[75] W. Surbeck, A. Bouthillier, and D. K. Nguyen, "Refractory insular cortex epilepsy: clinical features, investigation and treatment," Future Neurology, vol. 5, no. 4, pp. 491-499, 2010.

[76] W. Surbeck, A. Bouthillier, A. G. Weil et al., "The combination of subdural and depth electrodes for intracranial EEG investigation of suspected insular (perisylvian) epilepsy," Epilepsia, vol. 52, no. 3, pp. 458-466, 2011.

[77] W. Penfield and M. E. Faulk Jr., "The insula: further observations on its function," Brain, vol. 78, no. 4, pp. 445-470, 1955.

[78] P. Ryvlin, L. Minotti, G. Demarquay et al., "Nocturnal hypermotor seizures, suggesting frontal lobe epilepsy, can originate in the insula," Epilepsia, vol. 47, no. 4, pp. 755-765, 2006.

[79] M. Heers, S. Rampp, H. Stefan et al., "MEG-based identification of the epileptogenic zone in occult peri-insular epilepsy," Seizure, vol. 21, pp. 128-133, 2012.

[80] A. Afif, S. Chabardes, L. Minotti, P. Kahane, and D. Hoffmann, "Safety and usefulness of insular depth electrodes implanted via an oblique approach in patients with epilepsy," Neurosurgery, vol. 62, supplement 2, no. 5, pp. ONS471-ONS480, 2008.

[81] A. Bouthillier, W. Surbeck, A. G. Weil, T. Tayah, and D. K. Nguyen, "The hybrid operculo-insular electrode: a new electrode for intracranial investigation of peri-sylvian/insular refractory epilepsy," Neurosurgery. In press.

[82] H. Silfvenius, P. Gloor, and T. Rasmussen, "Evaluation of insular ablation in surgical treatment of temporal lobe epilepsy," Epilepsia, vol. 5, no. 4, pp. 307-320, 1964.

[83] H. Duffau, L. Capelle, M. Lopes, A. Bitar, J. P. Sichez, and R. van Effenterre, "Medically intractable epilepsy from insular low-grade gliomas: improvement after an extended lesionectomy," Acta Neurochirurgica, vol. 144, no. 6, pp. 563$573,2002$.

[84] J. Dobesberger, M. Ortler, I. Unterberger et al., "Successful surgical treatment of insular epilepsy with nocturnal hypermotor seizures," Epilepsia, vol. 49, no. 1, pp. 159-162, 2008.

[85] R. Malak, A. Bouthillier, L. Carmant et al., "Microsurgery of epileptic foci in the insular region," Journal of Neurosurgery, vol. 110, no. 6, pp. 1153-1163, 2009.
[86] Y. S. Park, Y. H. Lee, K. W. Shim et al., "Insular epilepsy surgery under neuronavigation guidance using depth electrode," Child's Nervous System, vol. 25, no. 5, pp. 591-597, 2009.

[87] M. R. Levitt, J. G. Ojemann, and J. Kuratani, "Insular epilepsy masquerading as multifocal cortical epilepsy as proven by depth electrode," Journal of Neurosurgery: Pediatrics, vol. 5, no. 4, pp. 365-367, 2010.

[88] M. von Lehe, J. Wellmer, H. Urbach, J. Schramm, C. E. Elger, and H. Clusmann, "Insular lesionectomy for refractory epilepsy: management and outcome," Brain, vol. 132, no. 4, pp. 1048-1056, 2009. 


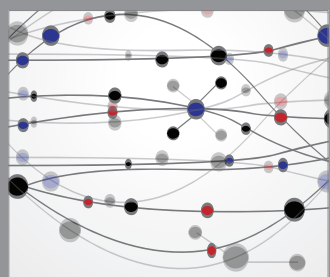

The Scientific World Journal
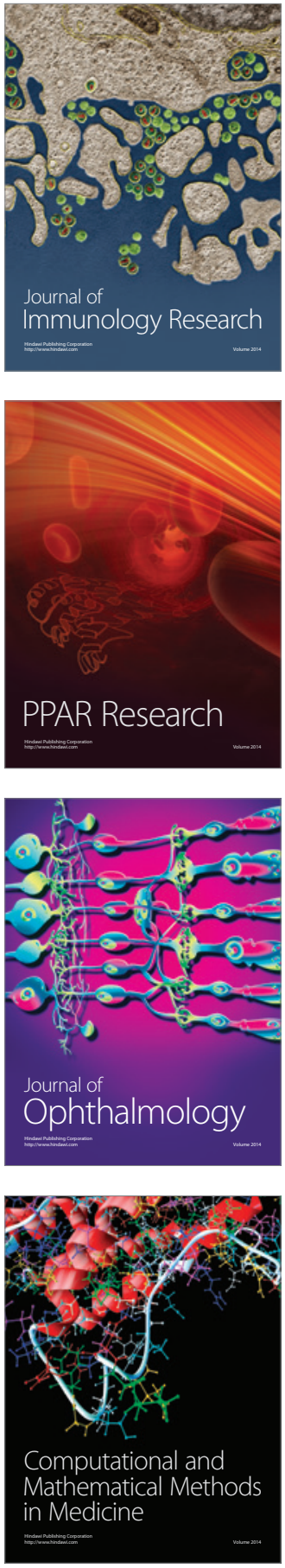

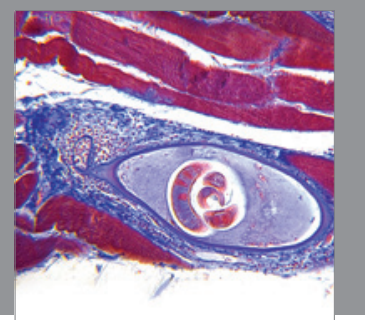

Gastroenterology

Research and Practice
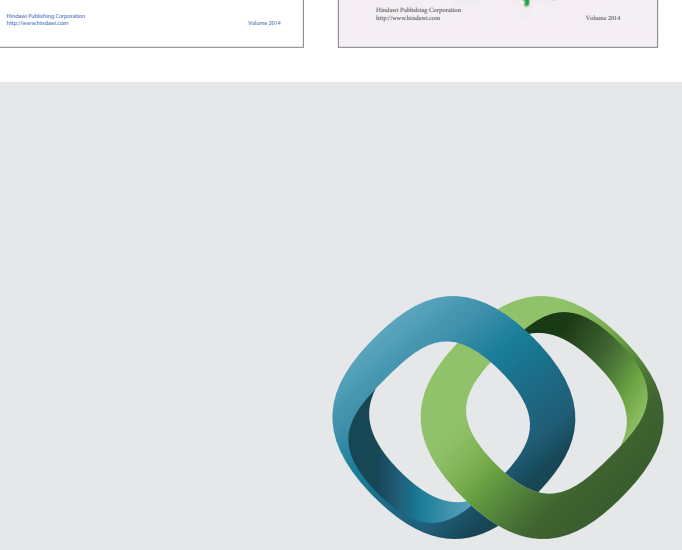

\section{Hindawi}

Submit your manuscripts at

http://www.hindawi.com
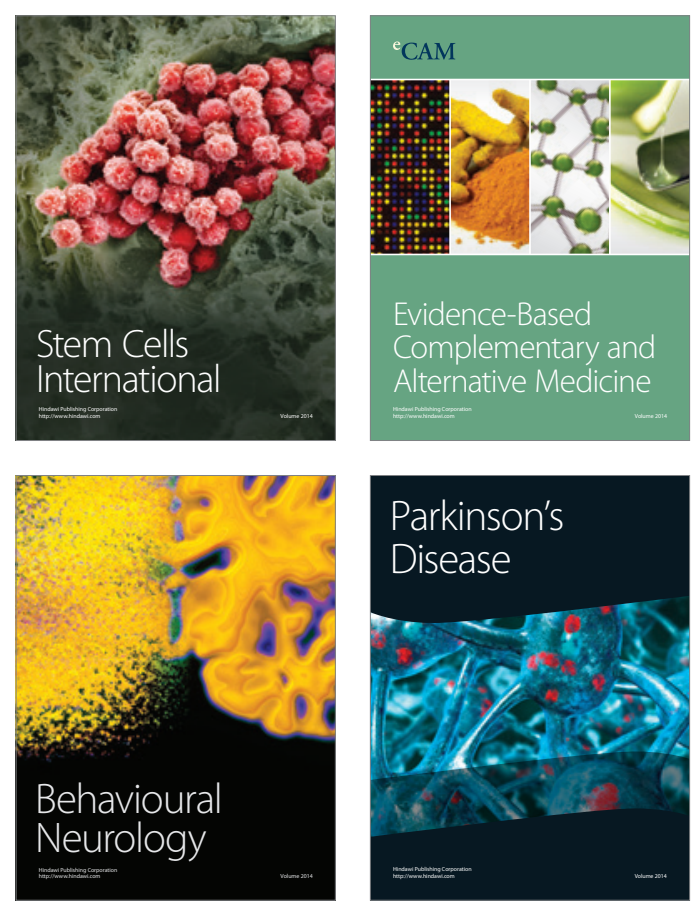

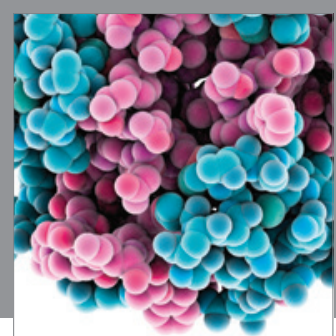

Journal of
Diabetes Research

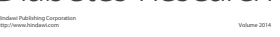

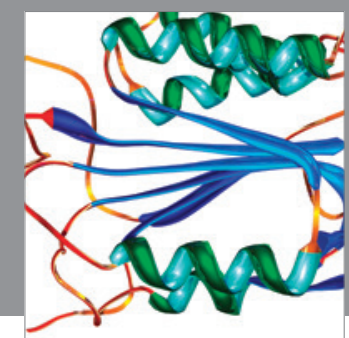

Disease Markers
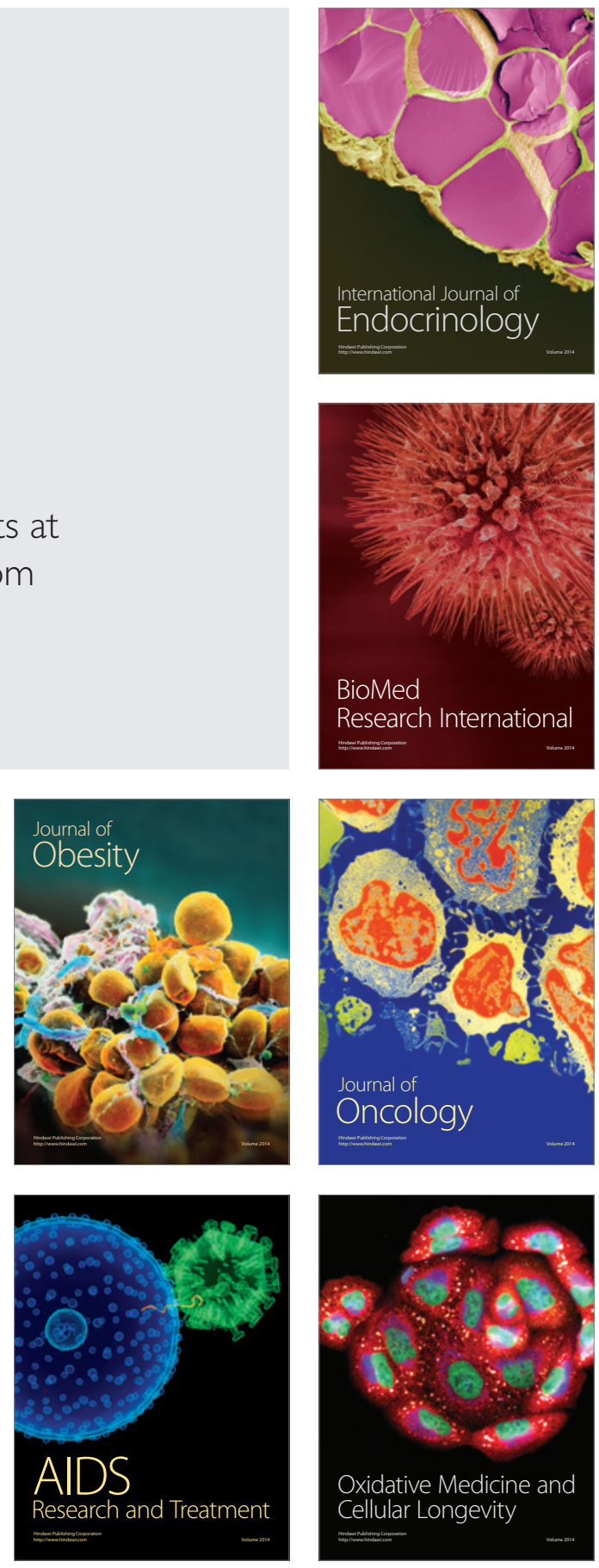